\title{
An Architecture for Source Code Protection
}

\author{
Noor Yasin, Abdul Ghafoor Abbasi, and Muhammad Shoaib
}

\begin{abstract}
Due to great competition in software industry the source code and binaries have to be protected. Source code available in plain form could be easily stolen and launched on any computer using IDE. We have given an architecture that will be used in both (single user environment and teaming environment) in order to provide source code protection. Source code protection would be achieved through authentication, authorization, encryption/decryption and hashing.
\end{abstract}

Index Terms-Encryption, authentication, authorization, hashing, source code SC, binaries, integrated development environment (IDE), authentication server \& ticket granting server (ATGS).

\section{INTRODUCTION}

In software industry, the most important asset of the industry is the source code of the software which is being developed. There is a lot of work that has been done in the secure communication, secure databases, and other security services in applications but source code itself is not being protected which might result in a great financial and data loss for an organization. Due to great competition in software Industry a Company coming up with new and unique ideas and then launch it in the market will in return generate great revenue. So in software industry idea and source code have a great importance.

Most of the software companies don't leak out their upcoming products until they are ready to be released in the market. Due to the importance of the idea and source code, source code theft is the biggest threat in software industry. Source code theft could be physical or via some botnet. In physical source code theft a person might get access to your system and copies the source files and takes it to his own system to recompile with the IDE. He got access to all of your work without any difficulty. The other case could be a Lab environment where many users have access to a common machine. In this situation any person logging on the system can view history in the IDE he could also access those source files if they are made available. In the second type where your competitors know the importance of your system might launch a botnet on your machine. In this way all your important files (including source files etc) could be stolen and sent to a remote machine. The person getting access to these source files might get a great benefit from it.

Our work is to protect these source files and binaries generated by the IDE using cryptographic technique. And if we don't protect the binaries, the binary could be reversed

Manuscript received October 15, 2014; revised January 5, 2015.

The authors are with National University of Sciences and Technology, Sector H-12, Islamabad, Pakistan (e-mail: 11msccsnyasin@ seecs.edu.pk, abdul.ghafoor@seecs.edu.pk,11msccsmshoaib@seecs.edu.pk). engineered to extract source code using some decompiler [1]. JAD could be used to launch a reverse engineering attack on binaries [2]. Some software's are available in the market that encrypts your files (including source files etc) but these files have to be encrypted manually every time. Due to this extra effort people just skip it. In our model source files and binaries would be automatically protected whenever the user exits the IDE. In this way the developer could pay his full attention on software development without caring about its protection. Our technique will provide confidentiality to source code and binaries, tempering of source code and idea leaking.

\section{RELATED WORK}

The idea of protecting source code and its binaries was influenced from some of its related work

1) Guy-Armand Yandji, Lui Lian Hao, Amir-Eddine Youssouf, Jules Ehoussou [3] presented a model for normal file encryption and decryption. The paper describes a methodology using AES and MD5 for encrypting files. The outcome file that will as a result be hashed and strongly encrypted through the software.

2) Xiufeng Zhang and Qiaoyan Wen [4] described the flexibility of Java language, which makes the protection become very difficult. Using decompiler [5] such as JAD we can easily extract the source code from the binary file. Therefore, any malicious user can use the anti-compiler tools to make reverse-engineering attacks. The paper presented an AOP-Based J2EE Source Code Protection technique in which they gave a solution to the problem that arises when encrypting J2EE applications.

3) ByungRae Cha [6] presented a CRYPTEX model for protecting software source code. The model presented protection and access control of software source code. The access control to the source code was achieved using digital certificate. The CRYPTEX consisted of software source code and an algorithm to control access.

4) A White Paper sponsored by CA Technologies [7] for Protecting API's against attack and hijack presented a secure API architecture. APIs are windows into an application and as with any window an API can easily be misused. APIs put application under the hacker microscope and increase attack surface on client application. So a solution was presented using SecureSpan API proxy.

5) SVN [8] and CVS [9] are used to control versions. A version control system keeps track of all work and all changes in a set of files, and allows several developers to access them. Access to these files is controlled using authentication and authorization if the files are not open source. Subversion can operate on network which will allow various people to modify and manage the same set of 
data.

\section{RISK FACTORS}

Source code is the main asset of an application, if somehow the source code is compromised the whole application/product get compromised. It should be necessary to become aware of the threats that are caused when source code or the binaries are compromised:

1) Source code can be used to steal the idea behind that product and can be used to make a similar product.

2) Source code can give attackers information about the working of your application and it will also provide him the loopholes in your application which would help him to launch attacks on your application.

3) Binary files can be revered engineered to generate source files using a decompiler and possible attacks could be launched by those source files.

4) By having the source code or binaries the attacker can add some unwanted feature to that product and make that malicious product available to the public.

5) Bypass license checks (patching) in your product and make a free copy available to public.

\section{APPLICATION DEVELOPMENT ENVIRONMENTS}

Source code could available to a single user or many users can have access to it. So our focus is on both types of source code protection environments

1) Single User Environment

2) Teaming Environment

\section{A. Single User Environment}

In a single user environment a single user using IDE on his system/computer would face all the challenges to the source code that we have discussed. The Source code is stored on the local drive in plain form. Anyone getting access to that drive can misuse or steal the source code. As the source code is in plain form the thief could launch it on his computer using an IDE without any extra effort. We have to protect this source code so that anyone getting access to your local drive won't have access to your source code.

\section{B. Proposed Solution}

Architecture is proposed for a single user environment to protect all the source code and binaries used by the IDE. Now the first step would be to authenticate the user using the IDE. So when the user launches the IDE a login screen will appear he would enter his credential (i.e. username and password) if his logging for the first time to that IDE he has to get registered and the hash of the password would be taken and stored with username at some secure place (i.e. database ). If his already registered the entered password has to be hashed and is compared with the hash that is already stored with a particular username. Now if the both hash match then the user would be login to the IDE.As you can see in Fig. 1 that after a successful login the IDE would lunch. If there is some kind of error the user would be asked to re-enter the credential, Skip and run IDE normally or exit.

Our main emphasis would be at start and exit of IDE.
Because on these two states the source code should be protected. When the user tries to open an existing project in IDE, The main protection module will ask the authorization module to check if he is authorized to view this project as shown in Fig. 3. The project would be decrypted with the user's key. If the key is correct the file would be decrypted without any error and make it available to user but if his the wrong guy he won't have access to it.

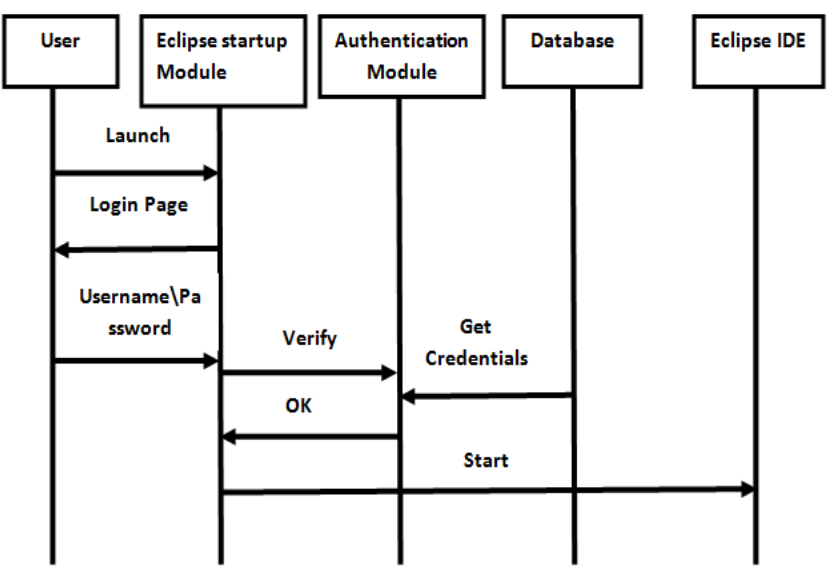

Fig. 1. Authentication sequence diagram.

As shown in Fig. 2 the authorization module will inform the protection main module that his the correct user. The protection main module will allow the user to view the file.

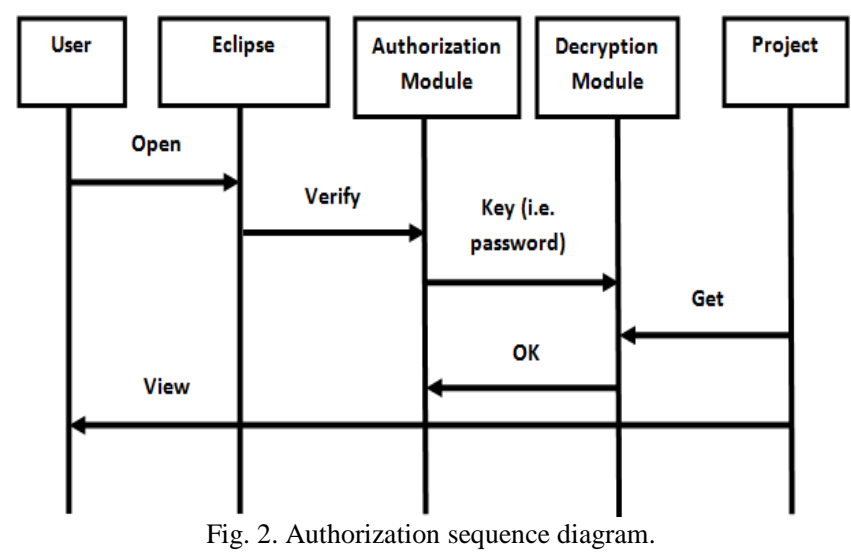

When the user tries to exit the IDE, first the source files would be saved automatically for any necessary changes then all the source files and binaries would be encrypted automatically with the existing user's key and then these encrypted source files and binaries would be stored on local drive.

Fig. 3 shows the complete architecture of single user source code protection environment. Authentication module has the task to authenticate the user. After authentication the IDE would be lunched. When opening a specific source code in this IDE the authorization module plays the role. This module checks whether the logged in user is authorized to view these files or not. The encryption and decryption modules would perform the encryption and decryption of the source code and binaries.

In the given architecture if someone gets access to your source code or binaries he won't be able to open them in an IDE, because these files are in encrypted form rather than plain form. 


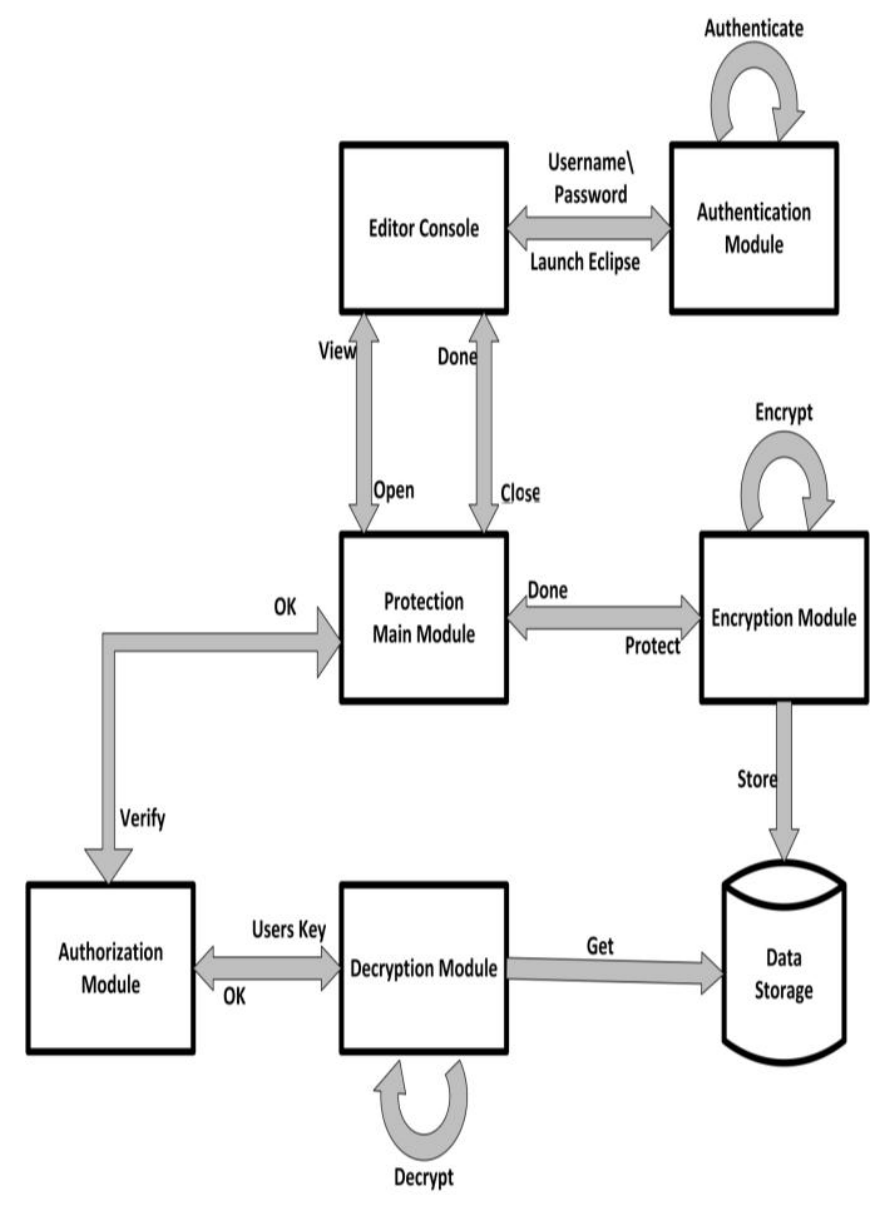

Fig. 3. Single user SC protection diagram.

\section{Implementation}

Eclipse is an open source IDE. We can get the complete source code from Git Repository. After getting and source code from Git Repository it was compiled and launched in eclipse IDE. So now eclipse was running its own source code. We had to find the right plugin that handled the starting and closing of eclipse IDE. The main class inside org.eclipse.equinox.launcher package was responsible to perform this task. AES was used for encryption and decryption of the source code. An authentication module was launched on startup having mysql database at backend and storing hashed passwords in it.

All these classes (i.e. authentication, authorization, encryption and decryption) were called inside main class. Now whenever the user launched the eclipse IDE the main class was revoked. Inside main class the authentication class has asked to check the authentication of user. Now when the user is authenticated user would try to open some project. When user tries to open any project the authorization classed is revoked. This class tries to decrypts all the .java and .class extension files with user's password. If all these files were encrypted by same user then he would be able to view them else an error would be launched that you're not authorized to view this project. When the user closes the eclipse IDE all the .java and .class files would be encrypted with users password.

\section{Teaming Environment}

The teaming environment is quite an interesting environment in which developers working on same project would like to have the source code to be centralized. They could fetch the source code to their own machine. There should be some mechanism that would authenticate and authorized users on this source code. The mechanism that we would present would allow user's authentication, authorization, uploading and downloading of the source code.

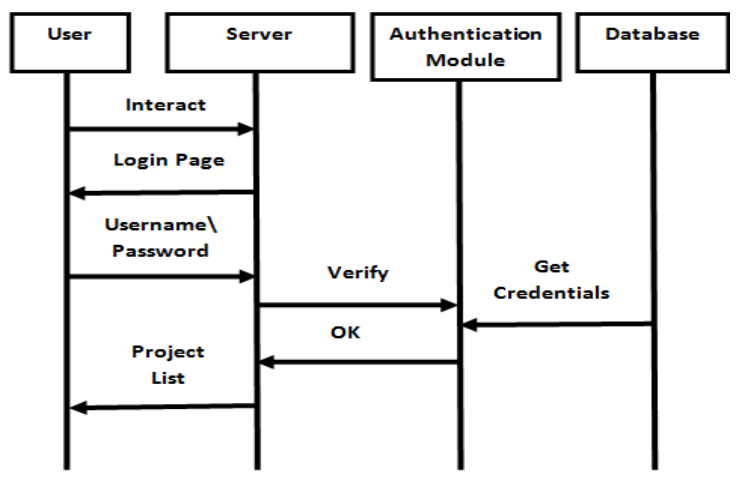

Fig. 4. Authentication sequence diagram.

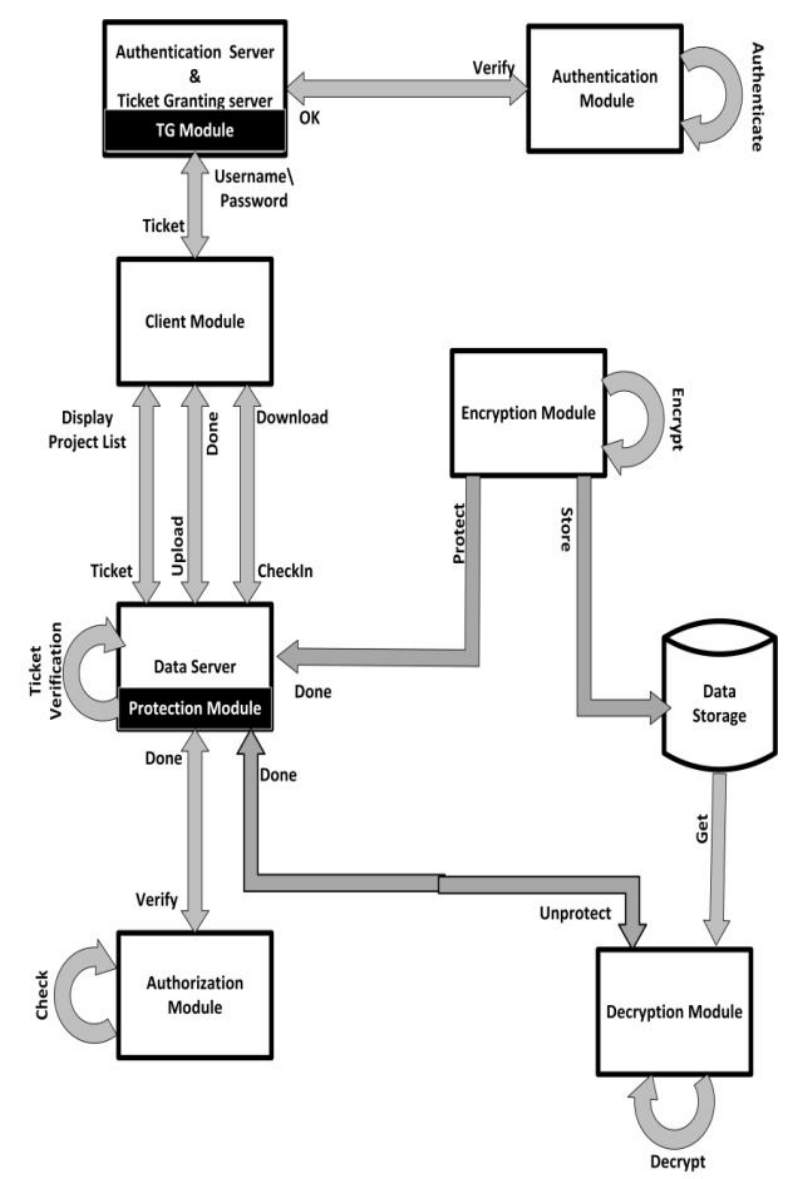

Fig. 5. Teaming environment SC protection diagram.

\section{E. Proposed Solution}

The teaming environment is an environment in which users would be working on a similar project and it has to be stored on some centralized location where everyone could have access to it. There would be a client module and server module to which the client would interact. User will add his credentials to the client module which would send it to the server module. As shown in Fig. 4 upon authentication of user the project list would be provided to the user.

Two servers would be needed to make this environment work (i.e. authentication/ TGS server and data server).First 
the client module will interact with the ATGS in order to achieve authentication. The client module will send username and password to the ATGS server after getting it from user.

The ATGS would have two modules running on it (i.e. ticket granting module \& authentication module) as shown in Fig. 5. The authentication module would verify that either the username or password provided are valid or not. The provided password would be taken hashed and compared with the one stored on the server, If they both match the user would be authenticated and then the ticket granting module would grant a ticket for the data server to the user. The user will take this ticket to the data server.

The ticket would be verified by the ticket verification module. If the ticket is valid then the user would be displayed with the list of all projects on the server shown in Fig. 4. He would be able to upload a project or download one if his authorized to do that. First let's consider the uploading of project. The user will upload a project and it would be encrypted using a shared key. The owner of the project can also provide the list of users that are authorized to download this project. He has to share the shared key with them.

Now in the downloading part the user would be authenticated the same way and his ticket is also verified as discussed earlier.

After seeing list of all projects the user would like to download one. The authorization module will check its authorization and if he is authorized then he would be asked to enter the shared key as shown in Fig. 6. Now using this shared key the project would be decrypted and downloaded onto user's machine. Authorization would also be checked when the user tries to open this project using IDE on his local machine.

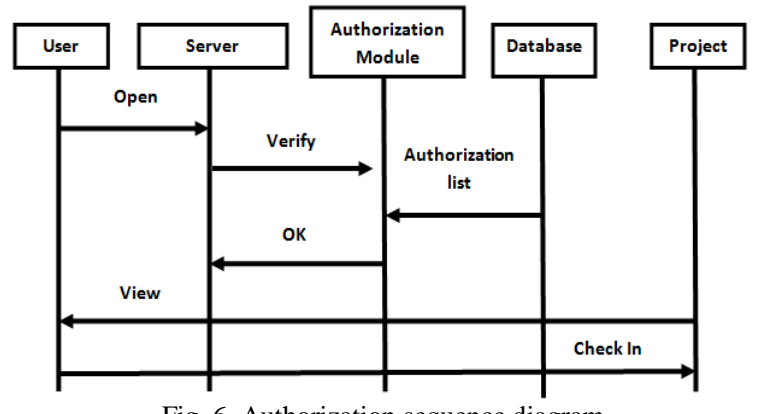

Fig. 6. Authorization sequence diagram.

\section{CONCLUSION}

Source code and the binaries have some great importance so they must be protected. The model presented in this paper gives a high level of protection to source code and binaries. It provides a mechanism in which the source code and binaries would be automatically encrypted by the IDE and saved rather than saving them in plain form. Only authorized users would have access to these source code and binaries. After verification of the authorized user the source code would be automatically decrypted and presented to the user. The teaming environment is also protected in which users would be authenticated and authorized to view or get their particular resource. When the source code gets too much the encryption and decryption process takes time. Future work could be done on speeding up this process by introducing a light weight encryption algorithm instead of AES.

\section{REFERENCES}

[1] T. Proesbsting, S. Watterson, and Krakatoa, "Decompilation in java (does bytecode reveal source?)," in Proc. the 3rd USENIX Conference on Object-Oriented Technologies and Systems, Portland, Oregon, 1997, pp. 185-197.

[2] Decompile Java Class File Using Decompilers. (May 2014). [Online]. Available:

http://viralpatel.net/blogs/decompile-class-file-java-decompiler-classjava-class/

[3] G. A. Yandji, L. L. Hao, A. E. Youssouf, and J. Ehoussou, "Research on a normal file encryption and decryption," presented at the International Conference on Computer and Management (CAMAN), Wuhan, China, 2011.

[4] X. Zhang and Q. Wen, "AOP-based J2EE source code protection," in Proc. International Conference on Computational Intelligence and Security Workshops, Harbin, Heilongjiang, China, 2007, pp. 581-584.

[5] T. Proesbsting, S. Watterson, and Krakatoa, "Decompilation in java (does bytecode reveal source?)," in Proc. the 3rd USENIX Conference on Object-Oriented Technologies and Systems, Portland, Oregon, 1997, pp. 185-197.

[6] B. Cha, "CRYPTEX model for software source code," presented at International Conference on Information Security and Assurance, Busan, Korea, April 24-26, 2008.

[7] Layer 7 Technologies. (May 2014). Protecting Your APIs against Attack \& Hijack. [Online]. Available: http://docs.media.bitpipe.com/io_11x/io_113161/item_826348/8\%20 -\%20Protecting\%20Your\%20APIs\%20Against\%20Attack\%20and\% 20Hijack.pdf

[8] Subversion. (May 2014). [Online]. Available: http://svnbook.red-bean.com/en/1.6/svn.intro.whatis.html

[9] CVS. (May 2014). [Online]. Available: http://en.wikipedia.org/wiki/Concurrent_Versions_System

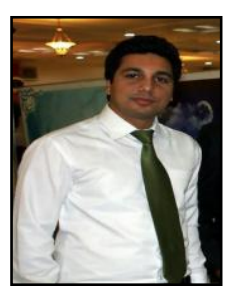

Noor Yasin completed his B.Eng. in software engineering from National University of Sciences and Technology (NUST) Pakistan in 2010 and he is now doing his $\mathrm{MS}$ in computer and communication security from National University of Science and Technology (NUST) Pakistan. Since 2012, he has been working as an assistant manager (IT) in an R\&D organization named Marine Systems (Pvt) Ltd. His research interests include software reverse engineering, information security, software development processes and methodologies. 